\title{
Automated 3D MRI Aortic Morphometry Demonstrates the Added Value of Volumes as Compared to Diameters
}

\author{
Thomas Dietenbeck ${ }^{1}$, Sophia Houriez--Gombaud-Saintonge ${ }^{1,2}$, Umit Gencer ${ }^{3}$, Alain Giron ${ }^{1}$, Gilles \\ Soulat $^{3}$, Elie Mousseaux ${ }^{3}$, Philippe Cluzel ${ }^{1,4,5}$, Alban Redheuil ${ }^{1,4,5}$, Nadjia Kachenoura ${ }^{1}$ \\ ${ }^{1}$ Sorbonne Université, CNRS, INSERM, Laboratoire d'Imagerie Biomédicale, LIB, Paris, France \\ ${ }^{2}$ ESME Sudria Research Lab, Paris, France \\ ${ }^{3}$ Hôpital Européen Georges Pompidou, Paris, France \\ ${ }^{4}$ Département d'imagerie cardiovasculaire DICVRIT, Institut de Cardiologie, Groupe Hospitalier \\ Pitié Salpêtrière APHP, Paris, France \\ ${ }^{5}$ Institute of Cardiometabolism and Nutrition (ICAN), Paris, France
}

\begin{abstract}
Aim: The diagnosis of thoracic aortic aneurysm is based on local aortic deformation associated to excessive aortic diameter (D). Maximal local aortic diameter was shown to be below the recommended surgical threshold in $30 \%$ of patients who ultimately developed aortic dissection. Aortic volumes integrate both dilation and elongation and may be more sensitive to changes in aortic geometry and less dependent on slice orientation and obliquity than diameter measurements. Methods: We studied 278 asymptomatic individuals with $3 D$ aortic MRI: 119 healthy volunteers $(h C), 53$ hypertensive patients (HT) and 106 patients with dilated ascending aorta of which 62 with tricuspid (APt) and 44 with bicuspid $(A P b)$ aortic valve. Automated $3 D$ aortic segmentation was performed and aortic lengths, maximal diameters and volumes were measured from sino-tubular junction to the brachiocephalic trunk for the ascending aorta (AAo) and from the left subclavian artery to the diaphragm for the descending aorta (DAo). Results: While AAo D increased by $40 \%$ between APt and HC, AAo volume increased by $170 \%$. Moreover, when comparing HT patients with controls, AAo volume difference was significant $(p<0.05)$ even after adjustment to BSA while AAo D was not. Conclusion: Aortic volumes measured automatically from $3 D$ MRI were able to characterize subclinical and pathological dilation more accurately than maximal diameters.
\end{abstract}

\section{Introduction}

One of the critical clinical features of aortic syndromes is that they are usually not perceived by apparently healthy individuals until a potentially lethal complication occurs such as aortic wall dissection or intramural hematoma. Imaging is widely recognized as the prime method to assess aortic diseases both for aneurysm detection and follow-up as well as prognostic evaluation and patient management decisions. However, despite the availability of 3D imaging techniques, prophylactic surgery remains indicated based on the 2D anatomic measurement of aortic diameters [1] which have been shown to fail in $30 \%$ of patients who finally develop aortic dissection [2]. It has also been shown that a significant elongation of the ascending aorta occurs in patients with thoracic aorta aneurysm and that excessive length is also an independent predictor of aortic dissection [3, 4].

Aortic volumes can be measured precisely and automatically from 3D MRI sequences [5] and we hypothesized that as they integrate both aortic dilation and elongation, they may be less hampered by slice orientation and obliquity and more sensitive to subclinical physiological and pathological changes in aortic geometry.

Thus, our objectives were to: 1) quantitatively characterize thoracic aorta morphology from 3D MRI, and 2) test the relevance of volumetric indices in the presence of aortic aneurysm as well as in hypertension, where only a mild dilation of the aorta is expected and in comparison to healthy individuals.

\section{Material and methods}

\subsection{Population and data acquisition}

We retrospectively included 119 healthy subjects (hC, mean age: $48 \pm 14$ years, 58 women), without overt cardiovascular disease, 53 hypertensive patients (HT, $52 \pm 12$ years, 26 women) and 106 consecutive patients with aortic root dilation and either tricuspid aortic valve (APt, $\mathrm{n}=62,65 \pm 12$ years, 15 women) or bicuspid aortic valve ( $\mathrm{APb}, \mathrm{n}=44,52 \pm 17$ years, 8 women). Patients with aortic aneurysm had no prior aortic surgery. All study participants gave their informed consent and the 

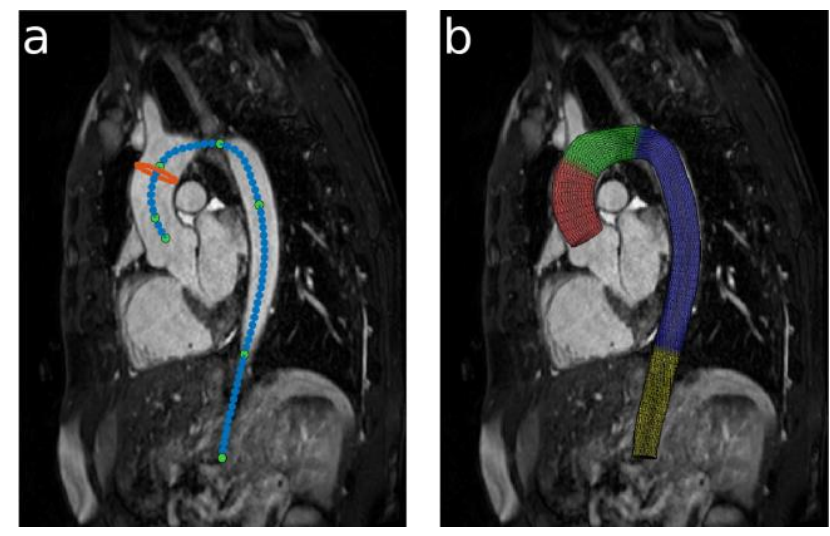

Figure 1: (a) Illustration of the BEAS cylindrical coordinates: anatomical landmarks (green dots) are used to initialize the interpolated the aortic centerline (blue dotted line). The aortic surface (red) then depends of the position and the angulation of the sliding plane along and perpendicular to the centerline. (b) Aortic surface and its division into 4 anatomical segments

study protocol was approved by the local ethics committee. All subjects underwent an MRI exam on either a $3 \mathrm{~T}$ magnet (GE Healthcare, $\mathrm{n}=117$ ) or a $1.5 \mathrm{~T}$ magnet (Siemens Healthcare, $\mathrm{n}=161$ ). 3D data were acquired during free breathing with ECG (diastole) and respiratory (expiration) gating in a sagittal oblique volume encompassing the thoracic aorta with the following scan parameters: GE: spoiled gradient echo SPGR, voxel size $=0.67 \times 0.67 \times 3.19 \mathrm{~mm}^{3}$, echo time $=$
$1.3 \mathrm{~ms}$, repetition time $=3.1 \mathrm{~ms}$ and flip angle $=24^{\circ}$; Siemens: steady state free precession SSFP, voxel size $=$ $0.66 \times 0.66 \times 1.13 \mathrm{~mm}^{3}$, echo time $=1.5 \mathrm{~ms}$, repetition time $=283 \mathrm{~ms}$ and flip angle $=90^{\circ}$.

\subsection{Aortic geometry}

3D MRI images were used for a precise evaluation of aortic morphology using a semi-automated segmentation method of the aorta (MIMOSA software [5], Laboratoire d'Imagerie Biomédicale, Sorbonne Université). After manually positioning 7 anatomical landmarks within the aorta (Figure 1a), the aortic centerline was interpolated using a spline function and an active contour method was used to segment the aorta. This technique represented the evolving boundary as an explicit function where the aortic radius was expressed as a function of the position and the angulation of a sliding plane along and perpendicular to the centerline.

After the segmentation, the initial rough centerline was automatically repositioned at the center of the aorta and the aorta was divided into 4 segments using the given anatomical landmarks (Figure 1b): ascending aorta (from the sino-tubular junction to the brachiocephalic trunk $\mathrm{BCT}$ ), aortic arch (from BCT to left subclavian artery LSA), descending aorta (LSA to diaphragm) and abdominal aorta (diaphragm to celiac trunk). In each segment, the following morphological indices were computed: centerline length, maximal diameters (perpendicular to the centerline) and volumes.

\begin{tabular}{|c|c|c|c|c|}
\hline & $\begin{array}{l}\text { Healthy controls } \\
\text { (hC) }\end{array}$ & $\begin{array}{c}\text { Hypertensives } \\
\text { (HT) }\end{array}$ & $\begin{array}{c}\text { Aneurysmal patients } \\
\text { with tricuspid valve } \\
(\mathrm{APt})\end{array}$ & $\begin{array}{c}\text { Aneurysmal patients } \\
\text { with bicuspid valve } \\
(\mathrm{APb})\end{array}$ \\
\hline $\mathrm{N}$ & 119 & 53 & 62 & 44 \\
\hline Gender (M/F) & $61 / 58$ & $27 / 26$ & $47 / 15$ & $36 / 8$ \\
\hline Age (y) & $48 \pm 14.3$ & $52.4 \pm 12.2$ & $64.7 \pm 12.3^{* * *}$ & $52.1 \pm 17.2$ \\
\hline $\operatorname{BSA}\left(\mathrm{m}^{2}\right)$ & $1.76 \pm 0.19$ & $1.84 \pm 0.22$ & $1.90 \pm 0.20^{* * * *}$ & $1.91 \pm 0.18^{* * *}$ \\
\hline $\mathrm{cSBP}(\mathrm{mmHg})$ & $112 \pm 13$ & $129 \pm 12^{* * *}$ & $122 \pm 15^{* *}$ & $117 \pm 16$ \\
\hline cDBP (mmHg) & $79 \pm 9$ & $88 \pm 9^{* * *}$ & $81 \pm 10$ & $80 \pm 11$ \\
\hline AAo L (mm) & $53.9 \pm 8.6$ & $58.3 \pm 8.4$ & $75.0 \pm 11.9^{* * * *}$ & $74.5 \pm 13.5$ \\
\hline AAo D (mm) & $28.8 \pm 3.8$ & $29.9 \pm 3.3$ & $41.3 \pm 5.1^{* * *}$ & $41.4 \pm 7.1^{* * *}$ \\
\hline AAo V (mL) & $32.7 \pm 12.5$ & $38.3 \pm 12.7^{*}$ & $89.4 \pm 30.0^{* * *}$ & $89.7 \pm 36.1^{* * *}$ \\
\hline AAo L / DAo L & $0.35 \pm 0.05$ & $0.40 \pm 0.06$ & $0.41 \pm 0.08^{* * *}$ & $0.43 \pm 0.07^{* * *}$ \\
\hline AAo D / DAo D & $1.27 \pm 0.13$ & $1.30 \pm 0.13$ & $1.44 \pm 0.21^{* * *}$ & $1.61 \pm 0.30^{* * *}$ \\
\hline AAo V / DAo V & $0.62 \pm 0.15$ & $0.74 \pm 0.18^{* * *}$ & $0.93 \pm 0.32^{* * *}$ & $1.25 \pm 0.52^{* * *}$ \\
\hline
\end{tabular}

Table 1: Subject characteristics for each group. BSA: Body surface area, cSBP / cDBP: central systolic / diastolic blood pressure, AAo: ascending aorta, DAo descending aorta, L: length, D: maximal diameter, V: volume.

$*$ means $\mathrm{p}<0.05, * * \mathrm{p}<0.01, * * * \mathrm{p}<0.001$ for comparisons against healthy controls. 


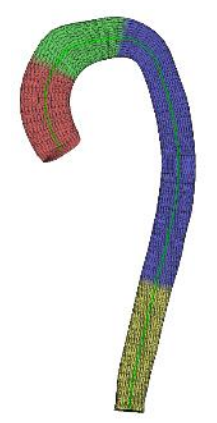

(a) hC

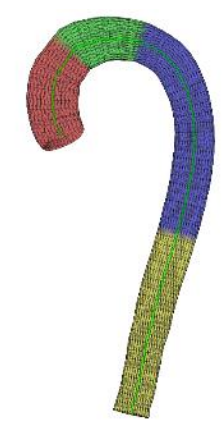

(b) $\mathrm{HT}$

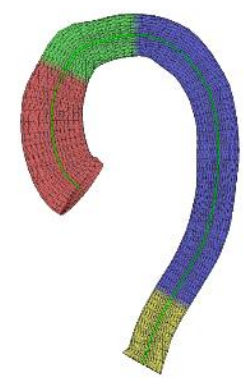

(c) APt

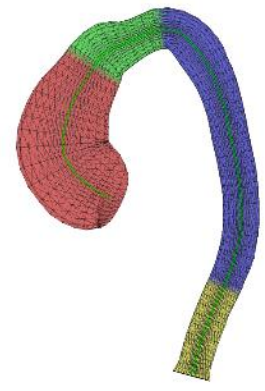

(d) $\mathrm{APb}$

Figure 2: 3D segmentation results illustrating morphological changes in the aorta across the studied groups: healthy control (a), hypertensive patient (b), aneurysmal patient with a tricuspid (c) or bicuspid (d) aortic valve.

\subsection{Statistical analysis}

Continuous variables were provided as means \pm standard deviations. An ANOVA was performed for comparisons across groups followed by t-test with Bonferroni correction to test the differences between the healthy controls and the 3 patient groups. All reported pvalues were two-sided and statistical significance was defined by a p-value $<0.05$.

\section{Results and discussion}

The 3D aortic segmentation of a single MRI dataset took less than 5 minutes in total on a standard computer including landmarks positioning and data loading time. Table 1 summarizes the basic characteristics of the population. Patient with a tricuspid aortic valve and an aneurysm of the ascending aorta were significantly older than the controls and had higher blood pressures. As expected, hypertensive patients also had higher blood pressures as compared to healthy controls.

\subsection{Morphological findings in the ascending aorta}

Figure 2 shows the segmented aorta of 4 male subjects matched for age illustrating morphological changes across groups. Figure 3 summarizes morphological indices (length, maximal diameter and volume) in the ascending aorta (AAo) over the 4 study groups.

First, the AAo volume was on average by $17 \%$ higher in hypertensives compared to healthy controls $(p<0.05$, Table 1). This volume difference can be explained by a significant elongation $(+8 \%, \mathrm{p}<0.01)$ and a more modest dilation $(+4 \%, \mathrm{p}=0.17)$. After indexation to $\mathrm{BSA}$, the difference in volume remained significant $(\mathrm{p}<0.05)$ while elongation $(\mathrm{p}=0.30)$ and dilation $(\mathrm{p}=0.91)$ were no longer significantly different. These findings highlight the ability of volumetry to identify subclinical morphological changes of the aorta by integrating information from the elongation and the homogeneous dilation of the ascending aorta associated with hypertension.

Second, in patients with AAo aneurysm, a highly significantly higher AAo volume was found compared to healthy controls (tricuspid aneurysmal patients (APt): $+174 \%, \mathrm{p}<0.001$; bicuspid aneurysmal patients $(\mathrm{APb})$ : $+175 \%, \mathrm{p}<0.001)$. This substantial difference in volume

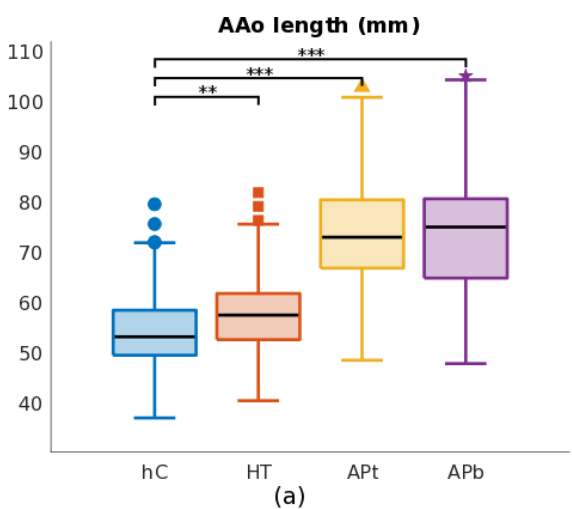

(a)

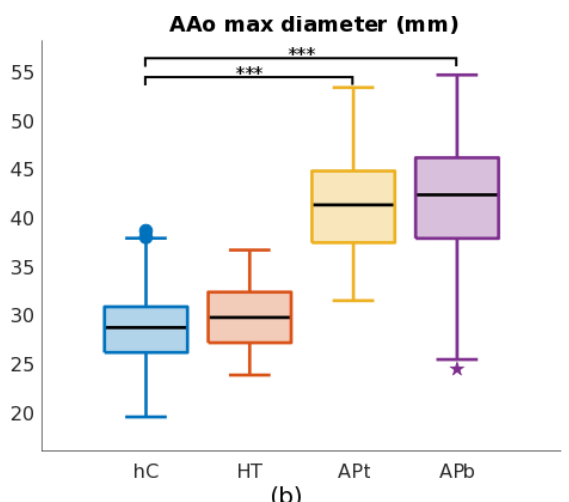

(b)

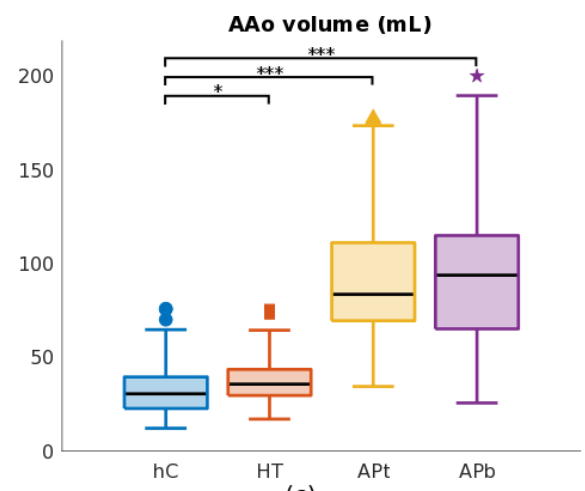

(c)

Figure 3: Aortic length (a), maximal diameters (b) as well as volumes (c) changes between healthy controls (hC), hypertensives (HT) and aneurysmal patients with a tricuspid (APt) or bicuspid (APb) aortic valve.

$*$ means $\mathrm{p}<0.05, * * \mathrm{p}<0.01$ and $* * * \mathrm{p}<0.001$. 
contrasted with a smaller increase in AAo maximal diameter (APt: $+44 \%, \mathrm{p}<0.001$;Pb: $+44 \%, \mathrm{p}<0.001$ ) as well as in AAo length (APt: $+39 \%, \mathrm{p}<0.001$; APb: $+38 \%, \mathrm{p}<0.001)$. These findings remained significant after indexation to BSA ( $\mathrm{p}<0.001)$. The markedly higher increase in AAo volume relatively to diameters may allow for better detection of aortic disease in at-risk patients and subsequently of better follow-up of aneurysmal progression.

Furthermore, in each group, all aortic measurements increased significantly with age in line with previous findings [6, 7]. Higher correlations with age were however obtained for volume than maximal diameter and length.

\subsection{Morphological changes in the ascending relative to the descending aorta}

Since the indexation of morphological cardiovascular indices to body size parameters has been widely debated in the literature, AAo morphological measures were indexed to the descending aorta (DAo) measures within the same patient (Table 1). Volumes AAo/DAo ratio changed significantly $(+20 \%, \mathrm{p}<0.001)$ in hypertensives when compared to healthy controls mainly due to differences in length ratio $(+15 \%, \mathrm{p}<0.001)$, since maximal diameters ratio remained unchanged $(+3 \%$, $\mathrm{p}=0.27)$. This highlights that in hypertension, while a homogeneous aortic dilation occurs in both the AAo and DAo, the aortic elongation primarily targets the AAo.

In aneurysmal patients, length, maximal diameter and volume AAo/DAo ratio increased significantly $(\mathrm{p}<0.001)$ reflecting a predominant AAo dilation and elongation. Furthermore, the differences observed in maximal diameter and volume ratio between $\mathrm{APt}$ and $\mathrm{APb}$ group were statistically significant $(\mathrm{p}<0.05)$ highlighting a higher dilation of the AAo in patients with a bicuspid aortic valve compared to patients with a tricuspid aortic valve.

\section{Conclusion}

In this study, we assessed aortic morphology using 3D MRI. We evaluated the ability of aortic length, maximal diameter and volume to characterize morphological aortic alterations in hypertension and aortic aneurysm. Aortic volume increases found in hypertension and aneurysmal aortic diseases were more substantial and independent of BSA as compared to increases in diameter. Indeed in addition to aortic dilation, volume also integrates elongation which was found to be significant in hypertension and ascending aorta aneurysm. The full clinical value of these morphological indices in the prediction of aortic dissection needs however to be demonstrated in large longitudinal studies.

\section{Acknowledgments}

We would like to acknowledge the ECOS-SUD project number A15S04 (France-Argentina) exchange grant and the FRM project ING20150532487 for funding part of the technical aspects of the study and the FRHTA for funding part of the acquisitions and clinical data generation.

\section{References}

[1] Erbel R, Aboyans V, Boileau C, et al. 2014 ESC Guidelines on the diagnosis and treatment of aortic diseases: Document covering acute and chronic aortic diseases of the thoracic and abdominal aorta of the adult The Task Force for the Diagnosis and Treatment of Aortic Diseases of the European Society of Cardiology (ESC). Eur Heart J 2014; 35: 2873--2926.

[2] Pape LA, Tsai TT, Isselbacher EM, et al. Aortic diameter $>$ or $=5.5 \mathrm{~cm}$ is not a good predictor of type A aortic dissection: observations from the International Registry of Acute Aortic Dissection (IRAD). Circulation 2007; 116: $1120-1127$.

[3] Krüger T, Oikonomou A, Schibilsky D, et al. Aortic elongation and the risk for dissection: the Tübingen Aortic Pathoanatomy (TAIPAN) project. Eur J CardioThorac Surg 2017; 51: 1119-1126.

[4] Heuts S, Adriaans BP, Gerretsen S, et al. Aortic elongation part II: the risk of acute type A aortic dissection. Heart 2018; 104: 1778--1782.

[5] Dietenbeck T, Craiem D, Rosenbaum D, et al. 3D aortic morphology and stiffness in MRI using semi-automated cylindrical active surface provides optimized description of the vascular effects of aging and hypertension. Comput Biol Med 2018; 103: 101-108.

[6] Kawel-Boehm N, Maceira A, Valsangiacomo-Buechel $\mathrm{ER}$, et al. Normal values for cardiovascular magnetic resonance in adults and children. J Cardiovasc Magn Reson 2015; 17: 29 .

[7] Adriaans BP, Heuts S, Gerretsen S, et al. Aortic elongation part I: the normal aortic ageing process. Heart 2018; 104: 1772-1777.

Address for correspondence:

Thomas Dietenbeck.

15 rue de l'école de médecine 75006, Paris. thomas.dietenbeck@ sorbonne-universite.fr 